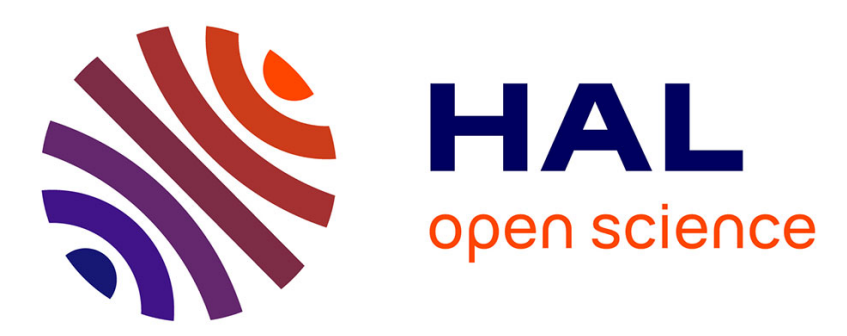

\title{
Enseignants non-titulaires dans le second degré en France : conditions de travail, bien-être professionnel et qualité de vie
}

T. Calon, S. Temam, M.-N. N Vercambre-Jacquot

\section{- To cite this version:}

T. Calon, S. Temam, M.-N. N Vercambre-Jacquot. Enseignants non-titulaires dans le second degré en France : conditions de travail, bien-être professionnel et qualité de vie. Archives des Maladies Professionnelles et de L'Environnement, 2020, 81 (2), pp.112-123. 10.1016/j.admp.2019.12.005 . hal02918428

\section{HAL Id: hal-02918428 \\ https://hal.science/hal-02918428}

Submitted on 20 Aug 2020

HAL is a multi-disciplinary open access archive for the deposit and dissemination of scientific research documents, whether they are published or not. The documents may come from teaching and research institutions in France or abroad, or from public or private research centers.
L'archive ouverte pluridisciplinaire HAL, est destinée au dépôt et à la diffusion de documents scientifiques de niveau recherche, publiés ou non, émanant des établissements d'enseignement et de recherche français ou étrangers, des laboratoires publics ou privés. 
ENSEIGNANTS NON-TITULAIRES DANS LE SECOND DEGRE EN FRANCE : CONDITIONS DE TRAVAIL, BIEN-ETRE PROFESSIONNEL ET QUALITE DE VIE

SECONDARY CONTRACTUAL TEACHERS IN FRANCE: WORKING CONDITIONS, PROFESSIONAL WELL-BEING AND QUALITY OF LIFE

Titre courant : Bien-être des enseignants non-titulaires du second degré

T Calon, S Temam, MN Vercambre-Jacquot

Fondation d'entreprise MGEN pour la Santé Publique

3 Square Max-Hymans

75748 Paris Cedex 15

Contact : mvercambre@mgen.fr

Keywords: teacher; precarious employment; job satisfaction; burnout; quality of life

Mots clés : enseignant ; emploi précaire ; satisfaction professionnelle ; épuisement professionnel ; qualité de vie 


\section{RESUME}

Objectifs Face à l'augmentation du nombre d'enseignants non-titulaires en France, notre objectif a été d'évaluer, dans le second degré, leurs conditions de travail et leur bien-être professionnel et général par comparaison aux enseignants titulaires.

Méthodes Dans l'enquête postale nationale «Qualité de Vie des Enseignants » (Fondation MGEN/Éducation nationale), 118 enseignants non-titulaires et 1298 enseignants titulaires du second degré ont été interrogés sur leur bien-être professionnel (satisfaction professionnelle, souhait de changer de profession, trois dimensions du Maslach Burnout Inventory) et général (questionnaire de qualité de vie WHOQOL-BREF). Après stratification sur le secteur d'enseignement (public/privé), ces indicateurs de bien-être ont été modélisés en fonction du statut d'emploi dans des modèles de régression ajustés sur de nombreux facteurs de confusion potentiels (âge, conditions d'exercice, etc.).

Résultats Les enseignants non-titulaires exerçaient dans des conditions globalement moins favorables que les titulaires dans les deux secteurs. Pourtant, dans le public, les non-titulaires $(n=70)$ présentaient de meilleurs indicateurs de bien-être professionnel que les titulaires $(n=1051)$ : ils souhaitaient moins souvent changer de profession $(\mathrm{OR}[95 \% \mathrm{IC}]=0,36[0,20 ; 0,66])$ et présentaient un épuisement émotionnel plus faible $(0,37[0,18 ; 0,78])$. Par contraste, les non-titulaires du privé $(n=48)$ tendaient à être moins satisfaits de leur expérience professionnelle et à avoir plus de symptômes d'épuisement émotionnel que leurs homologues titulaires $(\mathrm{n}=247)$. Concernant le bien-être général, les non-titulaires du privé étaient moins satisfaits de leur qualité de vie $(0,29[0,12 ; 0,66])$ que les titulaires. Enfin, les non-titulaires étaient significativement moins satisfaits de leur environnement de vie, qu'ils travaillaient dans un établissement public $(\beta[95 \% \mathrm{IC}]=-4,94$ points $[-8,17 ;-1,71])$ ou privé $(-6,33$ points $[-10,97 ;-1,69])$.

Conclusions Les enseignants non-titulaires du second degré privé présentaient de moins bons indicateurs de bien-être professionnel et général. Dans le second degré public, les résultats étaient plus contrastés, mais on retrouvait une moindre satisfaction vis-à-vis de l'environnement de vie. 


\section{SUMMARY:}

Purpose of the study: While the number of teachers with non-permanent contract ('contractual teachers') is steadily increasing in France, we aimed to characterize, in the secondary level, their working conditions and their general and professional well-being, in comparison with permanent teachers.

Method: In the population-based postal survey «Qualité de vie des enseignants » (MGEN Foundation/Ministry of Education), 118 secondary contractual teachers and 1298 secondary permanent teachers were asked about their professional well-being (job satisfaction, willingness to change job and the three dimensions of the Maslach Burnout Inventory), and general well-being (through the abbreviated version of the WHO-quality of life questionnaire). After stratification on type of school (state/private school), these well-being indicators were analyzed according to the employment status (contractual/permanent), using multivariate regression models adjusted on potential confounders (age, working conditions, etc.).

Results: Whatever the type of school, contractual teachers had less favorable work conditions than permanent teachers. However, and rather unexpectedly, in state schools, professional well-being indicators were better among contractual teachers $(n=70)$ compared to permanent teachers $(n=1051)$ : they were less likely willing to change job $(\mathrm{OR}[95 \% \mathrm{CI}]=0,36[0,20 ; 0,66])$ and to be emotionally exhausted $(0,37[0,18 ; 0,78])$. By contrast, in private schools, contractual teachers $(\mathrm{n}=48)$ tended to be less satisfied with their professional experience and to show more emotional exhaustion than permanent teachers $(\mathrm{n}=247)$. Regarding general well-being, contractual teachers in private schools were less satisfied with their quality of life $(0,29[0,12 ; 0,66])$. Lastly, contractual teachers were less satisfied about their life environment whether they worked in state $(\beta[95 \% \mathrm{CI}]=-4,94$ points $[-8,17$; $1,71])$ or private schools $(-6,33$ points $[-10,97 ;-1,69])$.

Conclusion: In secondary private schools, contractual teachers showed poorer indicators of professional and general well-being than permanent teachers. In secondary state schools, the results were more mixed, but there also, we observed a lower satisfaction with the living environment among contractual teachers. 


\section{INTRODUCTION}

Selon l'Organisation Internationale du Travail, l'emploi précaire se définit en référence à des critères tels que «l'incertitude sur sa durée, l'éventualité d'employeurs multiples ou d'une relation de travail déguisée ou ambiguë, l'absence d'accès à la protection sociale et aux avantages habituellement associés à l'emploi, une rémunération faible, et des obstacles juridiques et pratiques considérables pour adhérer à un syndicat et négocier collectivement »[1]. Plusieurs études épidémiologiques ont investigué les liens entre précarité de l'emploi, conditions de travail et santé. Ces études s'accordent sur le lien étroit entre emplois précaires et conditions de vie moins favorables, tant au travail- plus d'exposition aux risques physiques et chimiques, moins de latitude décisionnelle, moins de soutien social, moins d'accès à la médecine du travail [2,3] - que plus généralement - revenus plus bas [4], difficultés d'accès aux soins et/ou aux logements [5]. Par contraste, le lien retrouvé avec la santé physique ou mentale est plus fluctuant. Alors que certaines études montrent une association défavorable entre contrat précaire et certains indicateurs de santé ou de bien-être professionnel (indicateurs de santé tels que fatigue et troubles musculo-squelettiques [2,6], symptômes dépressifs [2-4,7-9], prise d'antidépresseurs [10], insatisfaction au travail [3] et présentéisme [2]), d'autres travaux nuancent cette relation $[4,11,12]$, des différences de champs et de méthodes sous-tendant sans doutes ces divergences.

Dans le secteur public, le statut de fonctionnaire assure une sécurité maximale de l'emploi. Bien qu'encore marginalement, d'autres types de contrats plus précaires existent cependant dans la fonction publique et ils tendent même à se développer ces dernières années, particulièrement dans le secteur de l'enseignement [13]. Pour devenir enseignant, dans le secteur public et privé sous contrat d'association qui dépendent tous deux du ministère de l'Éducation Nationale et de la Jeunesse, plusieurs voies sont possibles : sur concours - c'est la voie la plus courante - et ainsi devenir titulaire («fonctionnaire» dans le public, «maître contractuel» dans le privé) ou bien sans concours, mais selon certaines conditions : avoir un diplôme de niveau bac +3 - voire bac +2 depuis 2016 -, un diplôme technique ou une expérience professionnelle, et ainsi devenir enseignant «non-titulaire». Dans les faits, les 
enseignants non-titulaires sont recrutés en contrat de travail à durée déterminée de droit public (une année ou moins, reconductible) afin de pallier le manque d'effectifs des enseignants titulaires $[14,15]$.

Alors que les effectifs d'enseignants non-titulaires dans le secteur privé sous contrat sont stables, ils sont en augmentation depuis plusieurs années dans le secteur public, aussi bien dans le premier que dans le second degré [13]. Ainsi, en 2012-2013, ils étaient 958 non-titulaires dans le premier degré et 26403 dans le second degré; en 2017-2018, ils étaient respectivement 4092 et 39791 enseignants nontitulaires $[13,16]$. Concernant les conditions de travail, par comparaison aux titulaires, les enseignants non-titulaires ont des rémunérations moins importantes à temps de travail et ancienneté équivalents $[17,18]$, exercent plus souvent à temps partiel, enseignent dans des territoires moins attractifs et, spécifiquement dans le second cycle du second degré, plus souvent des disciplines professionnelles que des disciplines générales ou technologiques [13,15,16]. Par contraste avec ces données établies, le lien entre le statut d'emploi dans l'enseignement et la santé a fait l'objet de peu de travaux, dont aucun français. A notre connaissance, deux études, l'une italienne, l'autre espagnole, s'accordent sur le constat d'un moindre bien-être des enseignants ayant des contrats de travail temporaires (pouvant être comparés aux enseignants non-titulaires en France) $[19,20]$.

Devant l'ensemble de ces éléments, il nous a semblé intéressant d'étudier, en France, à partir de l'enquête nationale «Qualité de vie des enseignants », et en prenant comme référentiel les enseignants titulaires, les conditions d'exercice et le bien-être au travail et général des enseignants non-titulaires dans le second degré. Notre hypothèse de travail, en continuité avec la littérature disponible sur le sujet, était que, par comparaison aux enseignants titulaires, la santé des enseignants non-titulaires étaient moins bonne, la notion de "santé » étant comprise au sens large de «bien-être » de l'Organisation Mondiale de la santé [21]. Ce premier état des lieux visait à discerner des pistes de promotion de la santé des enseignants en France en fonction des spécificités liées à leur statut.

\section{METHODE}

Enquête "Qualité de vie des enseignants » 
L'enquête «Qualité de vie des enseignants » avait pour objectif principal de dresser un état des lieux du bien-être au travail des enseignants français. Cette étude épidémiologique élaborée par la Fondation MGEN pour la santé publique (www.fondationmgen.fr) [22], a été mise en œuvre en 2013 avec l'appui technique de la Direction de l'évaluation de la prospective et de la performance (DEPP) du Ministère de l'Éducation Nationale, de l'Enseignement Supérieur et de la Recherche. En pratique, 5000 enseignants ont été tirés au sort dans l'annuaire des personnels du ministère de l'Éducation nationale, après stratification sur le sexe, la classe d'âge, le type d'établissement et le secteur d'enseignement croisé avec l'appartenance à l'éducation prioritaire (public hors prioritaire / public prioritaire / privé sous contrat). Suite à une information postale et l'exclusion de 75 enseignants (71 avaient refusé de participer, 4 avaient indiqué être dans l'impossibilité de participer pour congés de longue durée), un questionnaire de 20 pages portant principalement sur les conditions de travail et la qualité de vie, a été envoyé directement dans l'établissement des enseignants. Parmi les questionnaires retournés, 2653 étaient exploitables, soit un taux de réponse brut de 54\%. Ces données auto-rapportées ont été complétées par des informations administratives (corps professionnel, modalité de service, caractéristiques de l'établissement d'affectation principale, épisodes de congés maladie, etc.) issues des bases de gestion des personnels de l'Éducation nationale et disponibles à la fois pour les répondants et les non-répondants à l'enquête (à l'exclusion des 71 refus).

L'ensemble du dispositif avait obtenu un avis favorable du Comité consultatif sur le traitement de l'information en matière de recherche dans le domaine de la santé (CCTIRS) et l'autorisation de la Commission nationale de l'informatique et des libertés (CNIL).

\section{Population d'étude}

Pour des raisons d'effectif, nous nous sommes focalisés sur les enseignants du second degré - ceux exerçant en collèges, lycées généraux et technologiques et lycées professionnels. En effet, au niveau national et sur l'année scolaire de l'enquête (2012-2013), les enseignants non-titulaires dans le premier degré étaient encore très peu nombreux dans le secteur public $(0,2 \%)$ [23]. De même, les enseignants non-titulaires dans le supérieur, référencés dans les bases ministérielles de gestion du personnel, 
représentaient à cette période moins de $1 \%$ des effectifs des enseignants du supérieur [23]. L'enquête «Qualité de vie des enseignants » reflétait ces chiffres, avec, parmi les répondants, seulement trois enseignants non-titulaires dans le $1^{\text {er }}$ degré et trois dans le supérieur. Au total, nous avons inclus dans la présente analyse 1416 enseignants du second degré ayant répondu à l'enquête, répartis en fonction de leur statut d'emploi : 1298 titulaires et 118 non-titulaires. Le groupe des non-titulaires incluait plusieurs catégories déclaratives : les "professeurs contractuels », les «maîtres auxiliaires » et les « vacataires », respectivement au nombre de 63, 50 et 5 dans notre échantillon.

\section{Indicateurs de bien-être}

a) Bien-être professionnel

Nous avons pris en considération cinq indicateurs de bien-être (ou de mal-être) professionnel évalués dans l'enquête «Qualité de vie des enseignants ». Le premier indicateur concernait la satisfaction professionnelle: «Aujourd'hui, si vous deviez faire le bilan de votre expérience professionnelle d'enseignant(e), vous diriez-vous... ?». Les 4 items de réponses ont été regroupés en deux catégories : positive (« assez satisfait(e) » ou « très satisfait(e) ») et négative (« assez peu satisfait(e) » ou «pas du tout satisfait(e) »).

Le second indicateur concernait le souhait de changer de profession: "Depuis que vous êtes enseignant(e), avez-vous déjà souhaité changer de profession?». Les réponses possibles étaient « Oui » ou « Non ».

Pour compléter ces questions ad-hoc, nous avons également examiné les trois dimensions du syndrome d'épuisement professionnel évalué par le Maslach Burnout Inventory adapté au métier d'enseignant (MBI - Educators Survey) [24]. Le MBI comporte 22 items répartis en trois dimensions :

1) l'épuisement émotionnel (score/54): impression de fatigue et de saturation affective et émotionnelle; 2) la dépersonnalisation (score/30): déshumanisation de la relation affective et représentation négative des élèves ; 3) le faible accomplissement au travail (score/48) : tendance à l'auto-évaluation négative de son travail, sentiment d'incompétence, de perte d'autorité, d'échec vis-à- 
vis de la réussite scolaire de ses élèves. Suivant l'approche de la symptomatologie du burnout préconisée par Maslach [24], les trois scores du MBI ont été dichotomisés en isolant le tertile «péjoratif » : épuisement émotionnel fort ( $\geq 27)$, dépersonnalisation forte $(\geq 5)$ et accomplissement professionnel faible $(<30)$.

b) Bien-être général

Pour évaluer le bien-être général, nous avons eu recours aux différents indicateurs issus du questionnaire abrégé de qualité de vie développé par l'Organisation Mondiale de la Santé (World Health Organisation - Quality of Life (WHOQOL-BREF))[25] et comprenant 26 items. Les deux premiers items généraux ont été étudiés en tant que tels : ils abordaient respectivement la qualité de vie ressentie («Comment trouvez-vous votre qualité de vie ?») et la santé subjective («Êtes-vous satisfait(e) de votre santé ?»). Les réponses pour chacun de ces deux items ont été regroupées en deux catégories positive/négative : «très bonne» ou «bonne» versus "ni bonne, ni mauvaise», «mauvaise », «très mauvaise » qualité de vie ; «très satisfait(e)» ou «satisfait(e) » versus «ni satisfait(e), ni insatisfait(e) », «pas satisfait(e)»ou «pas du tout satisfait(e) » de leur santé. Les 24 autres items venaient ensuite approfondir quatre dimensions majeures de la qualité de vie, avec pour chaque dimension un score systématiquement ramené sur 100:1) la santé physique : énergie et fatigue, douleur, dépendance, capacité de travail,..;2) la santé psychologique : sentiments négatifs et positifs, estime de soi, mémoire et concentration,...;3) les relations sociales : relations personnelles, support social,...; 4) l'environnement : ressources financières, sécurité, habitat, transport,...

\section{Interaction et stratification sur le secteur}

Une hétérogénéité de statut entre les non-titulaires du secteur public et du secteur privé sous contrat nous semblait probable étant donné les différences intersecteurs de barèmes de rémunération, de mode de recrutement et de recours au temps partiel $[15,18,23,26]$. Nous avons également émis l'hypothèse que le fait d'avoir exercé une autre profession dans le passé (indiquant une reconversion 
professionnelle) et le fait d'exercer à temps complet ou à temps partiel (engendrant des différences de revenus, du nombre d'heures d'enseignement, de la balance vie privée-vie professionnelle) pouvaient être source d'hétérogénéité de bien-être au sein même de la population des enseignants non-titulaires. Pour tester nos hypothèses d'hétérogénéité en fonction de ces trois facteurs d'interaction potentiels, nous avons respectivement mis en œuvre trois séries de modèles d'interaction, une série comprenant l'ensemble des modèles des indicateurs de bien-être en fonction du statut d'emploi, du facteur d'interaction potentiel, et du produit entre les deux. Ces analyses ont mis en évidence un effet d'interaction consistant pour les indicateurs de bien-être professionnel entre le statut d'emploi et le secteur d'enseignement mais pas avec les deux autres facteurs d'interaction testés (i.e. avoir exercé une autre profession dans le passé et la modalité de service). Par la suite, nous avons donc systématiquement stratifié nos analyses sur le secteur d'enseignement.

\section{Caractéristiques personnelles et professionnelles utilisées en variables d'ajustement}

Différentes caractéristiques personnelles et professionnelles des enseignants pouvant être liées à la fois au statut d'emploi et au bien-être ont été considérées comme facteurs de confusion potentiels : 1) des facteurs personnels d'ordre sociodémographique : sexe (homme / femme), catégorie d'âge (moins de 34 ans / 35-49 ans / plus de 50 ans) ; 2) des facteurs personnels caractérisant le travail : antécédents professionnels (avoir déjà exercé une autre profession : oui / non), modalité de service (temps complet / temps partiel), nombre d'établissement d'enseignement (un / deux ou plus), temps de trajet aller simple domicile-travail ( $\leq 15 \mathrm{~min} \mathrm{/} \mathrm{16-45} \mathrm{min} />45 \mathrm{~min}$ ), disciplines enseignées (disciplines fondamentales / technologiques-arts-sans spécialisation / éducation physique et sportive (EPS)), niveau d'enseignement $\left(1^{\text {er }}\right.$ cycle secondaire $/ 2^{\text {nd }}$ cycle secondaire général et technologique $/ 2^{\text {nd }}$ cycle secondaire professionnel); 3) des facteurs caractérisant l'établissement d'enseignement : secteur d'enseignement (public / privé sous contrat), taille de l'établissement ( $<600$ élèves / 600-999 élèves / $\geq 1000$ élèves), indicateur d'origine sociale majoritaire des élèves (plutôt favorisée / plutôt moyenne /

plutôt défavorisée). Ce dernier indicateur isole les quartiles extrêmes du ratio «proportion d'élèves d'origine plutôt favorisée / proportion d'élèves d'origine plutôt défavorisée » (données ministérielles 
disponibles au niveau de l'établissement) ; 4) des facteurs de risques psychosociaux au travail selon le Job Content Questionnaire (JCQ) développé par Karasek et enrichi par Karasek et Theorell [27] : scores de latitude décisionnelle, de demande psychologique et de soutien social au travail.

\section{Traitement des valeurs manquantes pour les variables d'ajustement}

Afin de maximiser la taille de l'échantillon d'analyse, et alors que le taux de valeurs manquantes pour chaque variable d'ajustement n'excédait pas 5\%, une imputation a été réalisée avant utilisation dans les modèles multivariés. Pour les facteurs d'ajustement catégoriels, les valeurs manquantes ont été imputées à la valeur la plus représentée ; pour les facteurs d'ajustement numériques (scores du JCQ essentiellement), elles ont été imputées par la moyenne. Une analyse de sensibilité dans l'échantillon des personnes sans données manquantes a été réalisée afin de s'assurer de la cohérence des résultats obtenus avec ou sans étape d'imputation.

\section{Analyses statistiques}

Dans un premier temps, nous avons comparé les caractéristiques sociodémographiques et professionnelles des enseignants du second degré non-titulaires à celles de leurs homologues titulaires en utilisant le test du Chi2 pour les variables catégorielles et le test de l'analyse de la variance (Fisher) pour les variables continues. Dans un second temps, nous avons étudié leurs différences de bien-être professionnel et de bien-être général au moyen de modèles de régressions logistiques pour les indicateurs dichotomiques et de modèles de régressions linéaires pour les indicateurs numériques. Pour chaque indicateur de bien-être, un premier modèle non ajusté (M1) permettait d'évaluer les différences «brutes » de bien-être. Un second modèle ajusté sur l'ensemble des facteurs de confusion potentiels permettait de raisonner à caractéristiques personnelles et professionnelles comparables. Les facteurs de confusion potentiels retenus étaient ceux associés $(\mathrm{p}<0,10)$ au statut d'emploi dans au moins un des deux secteurs d'enseignement. L'ancienneté, bien qu'associée au statut d'emploi, n'a pas été incluse dans les modèles multivariés pour éviter tout risque de sur-ajustement, avec l'âge et les 
antécédents professionnels, déjà considérés comme facteurs d'ajustement. D'autre part, bien que les scores de demande psychologique et de latitude décisionnelle du JCQ ne remplissaient pas le critère pour les retenir en tant que variable d'ajustement, ils ont été « forcés » dans le modèle multi-ajusté aux côtés de la troisième dimension du JCQ, le soutien social, qui elle remplissait le critère. Nous avons par ailleurs réalisé d'autres analyses de sensibilité : en excluant les professeurs agrégés, en excluant les temps partiels, et enfin en modélisant les scores du MBI en continu. Les analyses ont été faites à l'aide du logiciel STATA® SE 14.0.

\section{RESULTATS}

La population d'étude était composée de 1121 enseignants du second degré public (1051 titulaires et 70 non-titulaires) et de 295 enseignants du second degré privé sous contrat (247 titulaires et 48 nontitulaires).

Association entre le statut d'emploi et les facteurs sociodémographiques et professionnels, selon le secteur d'enseignement

Quel que soit le secteur d'enseignement (public / privé), les enseignants du secondaire étaient majoritairement des enseignantes, aussi bien chez les titulaires que les non-titulaires. Les non-titulaires étaient plus jeunes que les titulaires et étaient plus nombreux à avoir déjà exercé une autre profession par le passé (Tableau 1). Concernant leurs conditions d'exercice, comparativement aux titulaires, les non-titulaires exerçaient plus souvent à temps partiel, étaient plus nombreux à enseigner dans plusieurs établissements et mettaient plus de temps pour se rendre sur leur lieu de travail. Ils enseignaient plus fréquemment dans des disciplines d'enseignements «optionnels» (e.g. enseignement technique, artistique ou sans spécialité) et en second cycle professionnel, tout ceci face à des élèves d'origine sociale moins favorisée. Enfin, concernant les risques psychosociaux au travail, les non-titulaires rapportaient un soutien social au travail plus important que les titulaires, mais les différences n'étaient pas significatives concernant la latitude décisionnelle et la demande psychologique. 
Association entre le statut d'emploi et les indicateurs de bien-être au travail, selon le secteur d'enseignement

Concernant la satisfaction professionnelle, les non-titulaires du secteur privé tendaient à être moins satisfaits que les titulaires (Tableau 2), alors que dans le secteur public, la tendance était inverse (p d'interaction $<0.05$ ). Concernant le souhait de changer de profession, les non-titulaires du public étaient significativement moins nombreux à souhaiter changer de profession que les titulaires $(\mathrm{OR}[95 \% \mathrm{CI}]=0,36[0,20 ; 0,66])$, ce qui n'était pas retrouvé dans le privé ( $\mathrm{p}$ interaction $<0.05)$. Concernant la symptomatologie du burnout, les résultats différaient également entre le secteur public et le secteur privé. Dans le secteur public, les non-titulaires semblaient moins à risque que les titulaires pour l'ensemble des trois dimensions du syndrome d'épuisement professionnel, mais de manière significative seulement pour l'épuisement émotionnel $(\mathrm{OR}[95 \% \mathrm{CI}]=0,37[0,18 ; 0,78])$. Par contraste, dans le secteur privé, les non-titulaires tendaient à être plus à risque d'épuisement émotionnel (à la limite de la significativité).

Association entre le statut d'emploi et les indicateurs de bien-être général, selon le secteur d'enseignement

Dans le secteur public comme dans le secteur privé, la majorité des enseignants avaient une perception positive de leur qualité de vie. Ainsi, plus de six enseignants sur dix percevaient leur qualité de vie comme « bonne » ou « très bonne » (Tableau 3). La différence de qualité de vie entre titulaires et nontitulaires n'était pas significative dans le public mais elle l'était dans le secteur privé, en défaveur des non-titulaires $(\mathrm{OR}[95 \% \mathrm{CI}]=0,29[0,12 ; 0,66])$. Concernant la perception de leur état de santé, les réponses des enseignants étaient majoritairement positives (de l'ordre de 60\% également) quel que soit le statut d'emploi et le secteur d'enseignement et elles ne différaient pas significativement en fonction du statut d'emploi. Enfin, concernant les quatre dimensions de la qualité de vie évaluées par le WHOQOL-BREF, nous n'avons observé de différences significatives entre titulaires et non-titulaires 
que pour le score d'environnement, à la fois dans le secteur public et dans le secteur privé. Les nontitulaires rapportaient une moins bonne qualité de vie « environnementale » que les titulaires dans le public comme dans le privé $(\beta[95 \% \mathrm{IC}]=-4,94$ points $[-8,17 ;-1,71])$ et $-6,33$ points $[-10,97 ;-1,69]$ respectivement).

\section{Analyses de sensibilité}

Les résultats obtenus dans les différentes analyses de sensibilité entreprises (respectivement: restriction à l'échantillon des individus sans données manquantes pour toutes les variables y compris les facteurs d'ajustement; exclusion des professeurs agrégés; exclusion des temps partiels; modélisation des scores du MBI en continu) étaient cohérents avec les résultats principaux, quel que soit le secteur d'enseignement.

\section{DISCUSSION}

Dans une étude épidémiologique à caractère représentatif réalisée en 2013 auprès de 1416 enseignants du second degré, les enseignants non-titulaires exerçaient dans des conditions relativement moins favorables que les titulaires, et ce aussi bien dans le secteur public que dans le secteur privé sous contrat. Sur le plan du bien-être professionnel, la situation des non-titulaires était contrastée en fonction du secteur d'enseignement. Dans le secteur public, les deux différences significatives de bienêtre professionnel observées (sur cinq indicateurs) étaient, à l'encontre de notre hypothèse, plutôt à l'avantage des non-titulaires, ces derniers rapportant moins souvent un désir de changer de profession ainsi que moins de symptômes d'épuisement émotionnel que les enseignants titulaires, même après ajustement sur de nombreux facteurs de confusions potentiels. Dans le secteur privé, par contraste au secteur public, les enseignants non-titulaires étaient significativement moins satisfaits de leur expérience professionnelle que les titulaires. Ils tendaient également à présenter plus de signes d'épuisement émotionnel. Enfin, et ce aussi bien dans le secteur public que privé, les enseignants nontitulaires étaient globalement aussi positifs que leurs homologues titulaires quant à leur santé (globale, 
physique, psychologique et relationnelle), mais se déclaraient significativement moins satisfaits de leur environnement de vie.

Dans la présente étude, nous avons observé certaines spécificités dans les modalités d'exercice des enseignants non-titulaires qui vont dans le sens de conditions d'exercice moins favorables. En particulier, les enseignants non-titulaires sont amenés à travailler plus souvent dans plusieurs établissements scolaires, face à des élèves d'origine sociale moins favorisée, et leur temps de trajet est aussi plus long en moyenne. Cela est cohérent avec ce qui est retrouvé dans la littérature concernant les emplois temporaires, proposant globalement des conditions plus difficiles [2-4]. Concernant les indicateurs de bien-être, nos résultats, bien que contrastés en fonction du secteur d'enseignement, font également écho à ceux de la littérature chez les salariés en contrat temporaire, que ce soit dans le secteur privé [2-4,7-9] ou dans l'éducation [19,20], puisqu'un faisceau d'indices, particulièrement net pour les enseignants du privé, converge vers un moindre bien-être des enseignants non-titulaires.

Afin de comprendre les raisons de l'insatisfaction des non-titulaires vis-à-vis de leur environnement de vie, nous avons exploré les différences du score «environnement» du WHOQOL-BREF, item par item. Nous avons pu observer une satisfaction significativement moindre des non-titulaires quant à l'item «ressources financières » ainsi qu'à l'item «opportunités d'acquisitions d'informations et de connaissances », et ce de manière robuste à la fois dans le public et le privé (résultats non présentés dans un tableau/figure). Ces résultats sont en accord avec les données officielles concernant les rémunérations des enseignants puisque les non-titulaires ont des salaires moins élevés que les titulaires à temps de travail et ancienneté équivalents [17,18]. Quant à l'insatisfaction relative des enseignants non-titulaires vis-à-vis des opportunités d'acquérir des d'informations et des connaissances, elle renvoie possiblement au fait que ces enseignants, souvent plus jeunes et moins installés dans le métier, seraient plus en demande de formation et d'acquisition ou de développement de compétences que leurs homologues titulaires (diplômés). Alors que ces derniers s'estiment déjà insuffisamment formés par rapport à la moyenne européenne [28], cette insatisfaction accentuée des non-titulaires demande à être prise en compte, notamment dans le contexte actuel d'augmentation de leur effectif. 
Il est intéressant de noter que notre hypothèse initiale, celle d'un moindre bien-être des enseignants non-titulaires, a été invalidée en ce qui concerne la dimension professionnelle du bien-être, dans le secteur public tout au moins. De fait, les enseignants non-titulaires y présentaient de meilleurs indicateurs pour deux des cinq facettes du bien-être professionnel ici explorées, à savoir, le souhait de changer de profession et l'épuisement émotionnel. Un élément d'explication serait à trouver du côté des différences de parcours professionnel et de profils motivationnels en fonction du statut d'emploi. En effet, nous avons pu constater que les enseignants non-titulaires étaient beaucoup plus nombreux à avoir exercé une autre profession par le passé que les titulaires. De part cette expérience professionnelle antérieure plus courante, les non-titulaires auraient une vision plus large du monde du travail, et le métier d'enseignant y occuperait plus souvent la place privilégiée d'une reconversion positive (suite à une insatisfaction/difficulté dans le ou les poste(s) précédent(s)), voire d'un aboutissement professionnel, au-delà d'une rémunération en moyenne moins favorable $[17,18]$. Concernant plus spécifiquement la symptomatologie d'épuisement émotionnel plus faible chez les non-titulaires, d'autres facteurs pourraient également entrer en ligne de compte : en effet, les nontitulaires, du fait de leur ancienneté moindre (jusqu'en 2012, les CDD dans la fonction publique étaient la norme, de 3 ans maximum et renouvelables une fois, le véritable accès à la «CDIsation » des non-titulaires n'étant apparue qu'en 2012 (loi n 2012-347 du 12 mars 2012) [29]) seraient aussi moins impactés par les réformes qui touchent régulièrement le secteur de l'Education nationale [30]. Ils seraient ainsi moins à risque d'érosion professionnelle. Enfin, il est probable que parmi les non-titulaires, ceux qui restent seraient aussi ceux plutôt satisfaits et en bonne santé selon le principe du «travailleur sain ». Toutefois, les différences restent significatives après ajustement sur divers facteurs visant à capter de tels biais de confusion (âge, avoir déjà exercé une autre profession,...) suggérant que d'autres éléments sont à prendre en compte.

Dans le secteur privé, par contraste, et même si les associations n'atteignaient que rarement le degré de significativité dans notre échantillon relativement restreint, les indicateurs de bien-être professionnel étaient systématiquement moins bons chez les non-titulaires par comparaison aux titulaires, en accord avec notre hypothèse initiale. Ceci pourrait s'expliquer par un niveau de satisfaction plus élevé des 
enseignants titulaires du privé par rapport aux titulaires du public, autrement dit, la « référence de bien-être » par rapport à laquelle est comparée le bien-être des non-titulaires serait plus élevée dans le privé que dans le public. Une analyse à partir des données de la présente enquête [26] va en ce sens puisqu'elle montrait de meilleurs indicateurs de bien-être professionnel des enseignants du privé par rapport à leurs homologues du public. Un autre élément d'explication serait lié aux spécificités de recrutement des enseignants de l'enseignement privé sous contrat, qui est à 97\% confessionnel [31]. En effet, les enseignants titulaires du privé ont en général choisi le secteur privé comme lieu d'exercice, dans la mesure où le concours d'entrée qu'ils ont réussi, le CAFEP, est exclusif, contrairement au CAPES qui permet d'enseigner également dans le privé sous certaines conditions [32]. Les enseignants titulaires du privé sont donc peut-être particulièrement en phase avec leurs convictions sur leur lieu de travail, ce qui peut ne pas être le cas des non-titulaires dont la motivation première serait d'exercer le métier d'enseignant. Enfin, le ressenti plus défavorable des enseignants non-titulaires dans le privé pourrait provenir d'un sentiment plus marqué d'inégalité et de frustration lié à la certaine institutionnalisation du recours aux non-titulaires dans ce secteur.

\section{Forces et limites}

Parmi les forces de notre étude, nous pouvons retenir son originalité, puisqu'à notre connaissance, aucune étude française et très peu d'études internationales se sont intéressées aux conditions d'exercice et surtout au bien-être des enseignants non-titulaires par comparaison aux enseignants titulaires. Dans ce contexte, l'échantillonnage rigoureux de l'enquête était essentiel afin d'obtenir des résultats extrapolables à la population enseignante française. Par ailleurs, les informations administratives individuelles des bases ministérielles de gestion des personnels ont permis de compléter les réponses aux questionnaires, limitant les données manquantes pour les variables sociodémographiques et professionnelles. Enfin, l'utilisation d'outils épidémiologiques validés pour évaluer certains facteurs et événements d'intérêt (MBI, WHOQOL-BREF,...) appuie la fiabilité des résultats obtenus. 
Cependant, de par le caractère transversal de l'étude, il n'est pas possible d'interpréter les associations statistiques observées en termes de causalité ; autrement dit, les différences de bien-être significatives entre enseignants titulaires et non-titulaires ne sont pas forcément toutes imputables aux différences de statut. Néanmoins, comme nous avons pris soin d'ajuster sur de nombreux facteurs de confusion potentiels tels que l'âge et les modalités d'exercice, l'hypothèse d'un biais de confusion résiduel est affaiblie. Ensuite, la puissance de notre étude n'était pas optimale, compte tenu de la petite taille de notre échantillon de non-titulaires, en particulier suite à la stratification des analyses sur le secteur, stratification pourtant incontournable vu l'hétérogénéité des écarts titulaires/non-titulaires entre privé et public. Il serait intéressant de répliquer nos modèles dans des échantillons plus larges. Enfin, il est à souligner que les enseignants non-titulaires avaient moins bien participé à l'enquête que les titulaires (taux de réponse de $44 \%$ vs. $56 \%$ parmi les enseignants du second degré), ce qui n'est pas surprenant, étant donné l'envoi du questionnaire sur le lieu de travail et la plus grande instabilité professionnelle des non-titulaires. Une analyse spécifique a montré pour autant qu'il n'y avait pas d'interaction vis-àvis de la participation entre statut d'emploi et état de santé (appréhendé via l'indicateur «a eu un congé-maladie de plus de 3 jours dans l'année », renseigné à partir des données administratives, et donc disponible et fiable aussi bien pour les répondants que les non-répondants). Cela indique que le biais de participation des non-titulaires n'était probablement pas différentiel selon l'état de santé, et donc que, dans notre échantillon de répondants, l'estimation des associations entre indicateurs de santé et statut d'emploi étaient fiables.

\section{Préconisations}

Dans la mesure où les différences de bien-être professionnel des enseignants non-titulaires par rapport aux titulaires sont contrastées selon le secteur, il nous parait primordial de compléter les données disponibles et de croiser les regards, via la réalisation d'autres études, quantitatives mais aussi qualitatives ; il s'agira notamment de conforter nos observations et d'enrichir la compréhension de la réalité vécue par les enseignants non-titulaires, dans le public comme dans le privé. Il nous parait également pertinent de reprendre certaines des préconisations générales qui avaient été proposées dans 
un précédent travail plus spécifiquement centré sur le bien-être des enseignants en fonction de l'ancienneté [33] mais qui sont valables, selon nous, pour l'ensemble des enseignants, quel que soient le statut et le secteur d'enseignement. Une des préconisations était la mise en place de mesures de prévention collectives et individuelles pour lutter contre le mal-être au travail des enseignants, avec une prise en charge pluridisciplinaire et incluant l'ensemble des acteurs : direction de l'établissement d'enseignement, inspecteurs d'académie, médecins de prévention, psychologues, membres des Comités d'hygiène, de sécurité et des conditions de travail (CHSCT), assistants sociaux, collègues, etc. L'autre préconisation visait à réduire le déficit en médecins de prévention dans la fonction publique. Le recrutement de ces derniers était mis en avant, non seulement afin de respecter les obligations légales de suivi des enseignants mais également car les médecins de prévention, seuls à même de faire le lien entre santé et travail dans le cadre du secret médical et à pouvoir se rendre librement dans les établissements pour étudier les conditions de travail, représentent les véritables pivots dans la lutte contre la souffrance au travail. Afin d'appuyer l'action des médecins de prévention, et dans un contexte de manque structurel de profils adéquats, le renforcement de la pluridisciplinarité par recrutement d'autres acteurs de la prévention paraît également indispensable (infirmier(e)s en santé au travail, ergonomes, etc.) [34]. Enfin, certains dispositifs de prévention pour les personnels de l'Education nationale existent déjà (par exemple, les Réseaux PAS (Prévention, Aide et Suivi) [35]) et il nous semblerait opportun de les développer en collaboration avec les médecins de prévention et de les faire mieux connaitre.

\section{Conclusion}

Dans un contexte de désaffection du métier enseignant [36], alors que les effectifs des enseignants non-titulaires ne cessent de croître mais que le CDD reste le contrat principal parmi ces enseignants, il importe de mieux documenter et comprendre les causes de l'insatisfaction des enseignants nontitulaires, en particulier celui documenté ici dans le second degré privé sous contrat. 
Conflits d'intérêts : Les auteurs déclarent ne pas avoir de conflit d'intérêt en relation avec cet article

Remerciements : Les auteurs remercient Nathalie Billaudeau et Pascale Lapie-Legouis sans l'aide desquelles l'enquête et la présente analyse n'auraient pas pu être menées, ainsi que l'équipe du «Bureau des études statistiques sur les personnels » de la Direction de l'évaluation, de la prospective et de la performance (DEPP) du Ministère de l'éducation nationale. 


\section{BIBLIOGRAPHIE}

[1] Bureau international du Travail, Bureau des Activités pour les Travailleurs (ACTRAV). Du travail précaire au travail décent : document final du colloque des travailleurs sur les politiques et les réglementations destinées à lutter contre l'emploi précaire. 2012.

[2] Benach J, Vives A, Amable M, Vanroelen C, Tarafa G, Muntaner C. Precarious Employment : Understanding an Emerging Social Determinant of Health. Annu Rev Public Heal 2014;35:229-53.

[3] Lerouge L. Les effets de la précarité du travail sur la santé : le droit du travail peut-il s 'en saisir? Perspectives Interdisciplinaires Sur Le Travail et La Santé 2009:0-23.

[4] Directorate General for Internal Policies : Policy Department A : Economic and Scientific Policy. Precarious Employment in Europe: Patterns, Trends and Policy Strategy. 2016.

[5] Fondation Abbé Pierre pour le logement des défavorisés. Le logement et l'emploi, facteurs de précarisation mais aussi de développement. Rapport Annuel Sur l'état Du Mal-Logement En France 2014.

[6] Benavides FG, Benach J, Roman C. How do types of employment relate to health indicators? Findings from the Second European Survey on Working Conditions. J Epidemiol Community Heal 2000:494-501.

[7] Canivet C, Bodin T, Emmelin M, Toivanen S, Moghaddassi M, Östergren P. Precarious employment is a risk factor for poor mental health in young individuals in Sweden : a cohort study with multiple follow-ups. BMC Public Health 2016;16.

[8] Chun SY, Lee T-H, Kim W, Ju YJ, Kim T-H, Park E-C. Temporary work and depressive symptoms in South Korean workers. Occup Med (Chic Ill) 2017;67:421-4.

[9] Quesnel-vallée A, Dehaney S, Ciampi A. Temporary work and depressive symptoms : A propensity score analysis. Soc Sci Med 2010;70:1982-7. 
[10] Virtanen M, Kivima M, Ferrie JE, Elovainio M. Temporary employment and antidepressant medication : A register linkage study. J Psychiatr Res 2008;42:221-9.

[11] Santin G, Cohidon C, Goldberg M, Imbernon E, Santin G, Cohidon C, et al. Depressive symptoms and atypical jobs in France, from the 2003 Decennial health survey . Am J Ind Med 2009;52:799-810.

[12] Virtanen P, Janlert U, Hammarstro A. Exposure to temporary employment and job insecurity : a longitudinal study of the health effects. Occup Env Med 2010:570-4.

[13] Cour des comptes. Le recours croissant aux personnels contractuels : Un enjeu désormais significatif pour l'éducation nationale. 2018.

[14] Décret $n^{\circ} 2016-1171$ du 29 août 2016 relatif aux agents contractuels recrutés pour exercer des fonctions d'enseignement, d'éducation et d'orientation dans les écoles, les établissements publics d'enseignement du second degré ou les services relevant du ministre chargé de l'éducation nationale. Journal Officiel de la République Française 2016;n0202.

[15] Vocationenseignant.fr. Devenir enseignant contractuel ou vacataire : mode d'emploi. Visité Le 20/02/2019. https://vocationenseignant.fr/devenir-enseignant-contractuel-ou-vacataire-moded-emploi/.

[16] Direction de l'évaluation de la prospective et de la performance. Bilan social du ministère de l’Education Nationale et de la Jeunesse 2017-2018 - Enseignement scolaire. 2019.

[17] Defresne M. L'évolution du salaire des enseignants entre 2014 et 2015. Note d'information $\mathrm{N}^{\circ} 17.282017$.

[18] Defresne M. Les enseignants du public sont-ils mieux payés que ceux du privé ? Éducation et Formations 2016;92:33-56.

[19] Forcella L, di Donato A, Reversi S, Fattorini E, Boscolo P. Occupational stress, job insecurity and perception of the health status in italian teachers with stable or temporary employment. $\mathrm{J}$ Biol Regul Homeost Agents 2009. 
[20] Cladellas-Pros R, Castelló-Tarrida A, Parrado-Romero E. Satisfaction, health and work-related stress of the university professorship according to their contractual status. Rev Salud Pública 2018;20:53-9.

[21] Organisation mondiale de la Santé. Préambule à la Constitution de l'Organisation mondiale de la Santé, tel qu'adopté par la Conférence internationale sur la Santé, 1946.

[22] Billaudeau N, Gilbert F, Lapie-Legouis P, Vercambre-Jacquot M-N. Enquête "Qualité de vie des enseignants" : état des lieux. 16eme Colloq. l'ADEREST 2015 ; Arch. des Mal. Prof. l'Environnement, 2015, p. 391-2.

[23] Direction de l'évaluation de la prospective et de la performance. Bilan social du ministère de l’Education Nationale 2012-2013, partie I - Ministère de l'éducation nationale. 2013.

[24] Maslach C, Jackson S, Leiter M. Maslach Burnout Inventory Manual. Consult Psychol Press Inc 1996.

[25] Baumann C, Erpelding ML, Regat S, Collin JF BS. Le questionnaire de qualité de vie WHOQOL-BREF : valeurs de références françaises des dimensions santé physique, santé psychologique et relation sociale. Rev d'epidemiologie Sante Publique 2010;58:33-9.

[26] Billaudeau N, Vercambre-Jacquot M-N, MGEN. Satisfaction professionnelle des enseignants du secondaire : Quelles différences entre public et privé ? Éducation et Formations 2015;8889:201-20.

[27] Karasek R, Theorell T. Healthy Work : Stress, Productivity, and the Reconstruction of Working Life. Basic Books, New York 1990.

[28] Charpentier A, Solnon A. La formation continue, un levier face à la baisse du sentiment d'efficacité personnelle des enseignants au collège ? Note d'information ํ⒚23 2019 .

[29] Loi n 2012-347 du 12 mars 2012 relative à l'accès à l'emploi titulaire et à l'amélioration des conditions d'emploi des agents contractuels dans la fonction publique, à la lutte contre les discriminations et portant diverses dispositions relatives à la fonction publique. Journal Officiel de la République Française 2012;n0062:page 4498. 
[30] Ministère de l'Éducation nationale et de la Jeunesse. L'histoire des grands textes de l’Éducation nationale 2017. https://www.education.gouv.fr/cid102199/-frise-interactive-1histoire-des-grands-textes-de-l-education-nationale.html.

[31] Enseignement catholique de Paris. Grands chiffres de l'enseignement catholique 2019. http://www.ec75.org/index-5-8306--.html.

[32] Uher C. Quelle différence entre les enseignants du public et les professeurs du privé ? 2011. https://www.ifrap.org/education-et-culture/quelle-difference-entre-les-enseignants-du-publicet-les-professeurs-du-prive.

[33] Zavidovique L, Gilbert F, Vercambre-Jacquot M-N. Bien-être au travail et qualité de vie des enseignants : quelles différences selon l'ancienneté ? Arch Des Mal Prof l'Environnement 2017;760:641-96.

[34] Inspection Générale de l'Administation, Inspection Générale des Affaires Sociales, Inspection Générale de l'administration de l'éducation nationale de l'enseignement supérieur et de la recherche. Rapport sur la médecine de prévention dans les trois fonctions publiques. 2014.

[35] Ministère de l'éducation nationale, de l'enseignement supérieur et de la recherche. Bulletin officiel 2004;n³ 36 :page 2084.

https://www.education.gouv.fr/bo/2004/36/MENA0402174X.htm.

[36] Valette C. Concours enseignants 2018 du second degré public. Note d'information $N^{\circ} 19.25$ 2019. 
Tableau 1 : Comparaison des enseignants titulaires/non-titulaires dans le second degré, stratifiée selon le secteur d'enseignement

\begin{tabular}{|c|c|c|c|c|c|c|}
\hline & \multicolumn{3}{|c|}{ Secteur public } & \multicolumn{3}{|c|}{ Secteur privé sous contrat } \\
\hline & $\begin{array}{l}\text { Titulaires } \\
(\mathrm{N}=1051)\end{array}$ & $\begin{array}{c}\text { Non- } \\
\text { titulaires } \\
(\mathrm{N}=70)\end{array}$ & $p^{1}$ & $\begin{array}{l}\text { Titulaires } \\
(\mathrm{N}=247)\end{array}$ & $\begin{array}{c}\text { Non- } \\
\text { titulaires } \\
(\mathrm{N}=48)\end{array}$ & $p^{1}$ \\
\hline \multicolumn{7}{|l|}{ Caractéristiques sociodémographiques } \\
\hline Sexe (\%) & & & 0,22 & & & 0,38 \\
\hline Homme & 37 & 44 & & 27 & 33 & \\
\hline Femme & 63 & 56 & & 73 & 67 & \\
\hline Age (\%) & & & 0,01 & & & $<0,01$ \\
\hline$<35$ ans & 18 & 29 & & 10 & 35 & \\
\hline $35-49$ & 51 & 57 & & 47 & 56 & \\
\hline$\geq 50$ & 31 & 14 & & 43 & 8 & \\
\hline \multicolumn{7}{|l|}{ Facteur professionnels personnels } \\
\hline $\begin{array}{l}\text { Autre profession exercée par le } \\
\text { passé (\%) }\end{array}$ & & & $<0,001$ & & & $<0,001$ \\
\hline Oui & 30 & 76 & & 43 & 77 & \\
\hline Non & 70 & 24 & & 58 & 23 & \\
\hline Modalité de service (\%) & & & 0,27 & & & 0,02 \\
\hline Temps complet & 86 & 81 & & 83 & 69 & \\
\hline Temps partiel & 14 & 19 & & 17 & 31 & \\
\hline Nombre d'établissement (\%) & & & $<0,001$ & & & 0,02 \\
\hline 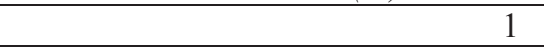 & 91 & 73 & & 89 & 77 & \\
\hline$\geq 2$ & 9 & 27 & & 11 & 23 & \\
\hline Temps de trajet aller simple (\%) & & & 0,001 & & & $<0,001$ \\
\hline$\leq 15 \mathrm{~min}$ & 40 & 21 & & 36 & 19 & \\
\hline $16-45 \mathrm{~min}$ & 46 & 50 & & 49 & 40 & \\
\hline$>45 \mathrm{~min}$ & 14 & 29 & & 14 & 42 & \\
\hline Disciplines enseignées ${ }^{2}(\%)$ & & & 0,001 & & & 0,59 \\
\hline Techno-Art-Sans spécialisation & 20 & 39 & & 19 & 25 & \\
\hline $\begin{array}{r}\text { Fondamentales } \\
\end{array}$ & 69 & 59 & & 74 & 69 & \\
\hline EPS & 11 & 3 & & 7 & 6 & \\
\hline Niveau d'enseignement (\%) & & & 0,003 & & & 0,02 \\
\hline 1er cycle secondaire & 50 & 57 & & 51 & 42 & \\
\hline 2nd cycle général/technologique & 32 & 14 & & 33 & 25 & \\
\hline 2nd cycle pro & 18 & 29 & & 16 & 33 & \\
\hline \multicolumn{7}{|l|}{ Caractéristiques de l'établissement } \\
\hline Taille établissement (\%) & & & 0,08 & & & 0,08 \\
\hline$<600$ élèves & 44 & 56 & & 43 & 60 & \\
\hline 600-999 élèves & 32 & 30 & & 24 & 17 & \\
\hline$\geq 1000$ élèves & 24 & 14 & & 33 & 23 & \\
\hline Origine sociale des élèves ${ }^{3}(\%)$ & & & 0,002 & & & 0,01 \\
\hline Milieu favorisé & 19 & 9 & & 49 & 40 & \\
\hline Milieu moyen & 52 & 44 & & 45 & 40 & \\
\hline Milieu défavorisé & 29 & 47 & & 6 & 21 & \\
\hline \multicolumn{7}{|l|}{ Risques psycho-sociaux au travail } \\
\hline \multicolumn{7}{|l|}{$\begin{array}{l}\text { Scores du Job Content } \\
\text { Questionnaire [moyenne (ET)] }\end{array}$} \\
\hline Demande psychologique (/36) & $23,9(3,9)$ & $23,4(3,8)$ & 0,31 & $23,4(4,3)$ & $23,1(3,9)$ & 0,65 \\
\hline Latitude décisionnelle (/96) & $75,7(8,3)$ & $75,8(8,0)$ & 0,94 & $76,7(7,7)$ & $76,3(6,7)$ & 0,77 \\
\hline Soutien social (/32) & $22,5(3,7)$ & $23,5(3,9)$ & 0,04 & $23,3(4,1)$ & $24,1(4,1)$ & 0,18 \\
\hline \multicolumn{7}{|c|}{ 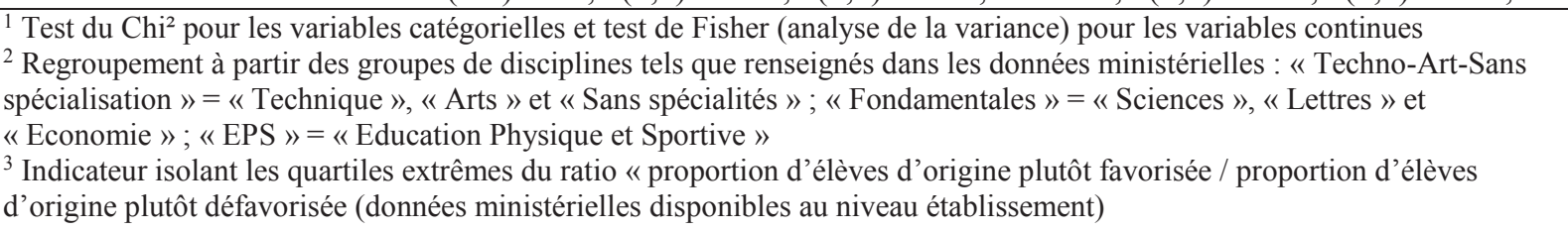 } \\
\hline
\end{tabular}




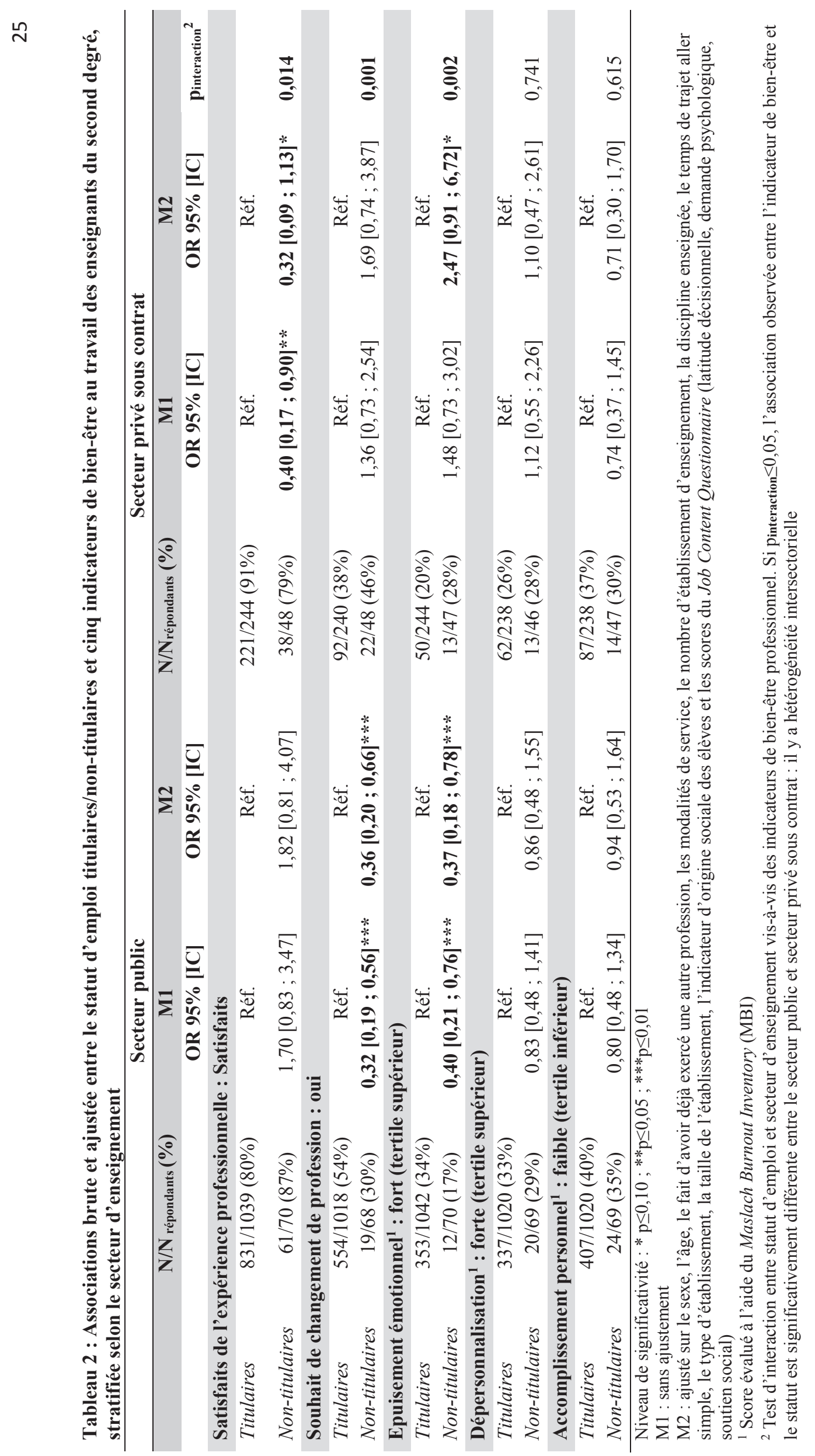




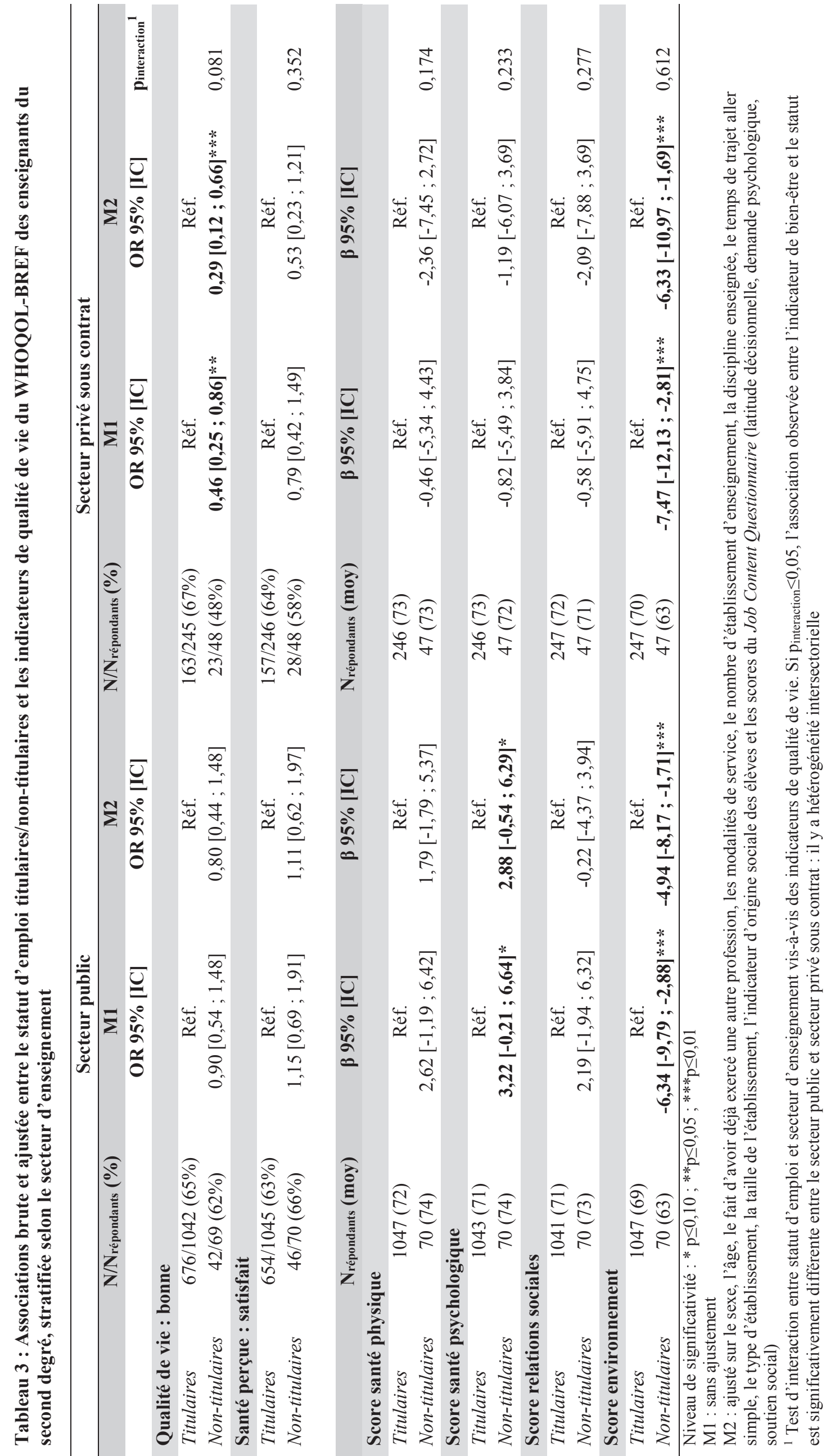

